\title{
A New Approximate Solution for Scattering by Thin Dielectric Disks of Arbitrary Size and Shape
}

\author{
Il-Suek Koh, Member, IEEE, and Kamal Sarabandi, Fellow, IEEE
}

\begin{abstract}
In this paper, an approximate solution for electromagnetic scattering by a very thin planar homogeneous dielectric object is presented. This solution is obtained from a volumetric integral equation using Fourier transform and is shown to be uniformly valid from low to high frequencies at all incidence angles including edge-on incidence. Validity of the solution is demonstrated through a comparison with canonical objects such as an infinite dielectric slab, and a number of two-dimensional (2-D) and three-dimensional (3-D) dielectric scatterers. For 2-D, and 3-D scatterers, the approximate solution is compared with a method of moments solution. In all cases examined the approximate formulation provides very accurate results except for situations where the dielectric constant is very high.
\end{abstract}

Index Terms-Scattering, thin dielectric disk.

\section{INTRODUCTION}

$\mathbf{S}$ CATTERING from thin dielectric objects is a classic research topic in electromagnetics which has found a number of useful applications. A traditional approach is to model thin dielectrics with resistive sheets, and for several canonical geometries of resistive sheet bodies, exact solutions are found [1]. For example, solutions for scattering from a resistive half-plane was obtained using the Wiener-Hopf technique or dual integral equation formulation, which are represented in terms of Maliuzhinets half-plane function. For other geometry such as a finite strip or a disk exact solutions are not yet known [2]. Therefore, for arbitrary structures, a numerical method such as method of moments (MoM) or approximate analytical solutions such as physical optics (PO) or physical theory of diffraction (PTD) which includes the effect of edge diffraction are applied.

A thin dielectric disk is widely adopted to model broad leaves of deciduous trees [3], [4]. To accurately estimate characteristics of forest channel such as backscattering, attenuation, etc. over a wide range of frequencies, it is very important to increase the accuracy of scattering formulations for the constituent scatterers [5], . Currently two approximate scattering solutions, volumetric integral physical optics (VIPO) and Rayleigh-Gans, are commonly used. However, the accuracy of these two approximations is strongly influenced by the size and thickness of the disk, frequency, dielectric constant, and the incidence angles [5]. Rayleigh-Gans is valid when the thickness is sufficiently small $\left(\left|k_{0} t \sqrt{\varepsilon_{r}-\sin \theta_{i}}\right| \ll 1\right.$, see Fig. 1). VIPO can generate accu-

Manuscript received January 5, 2004; revised October 11, 2004.

I.-S. Koh is with the Graduate School of Information Technology and Telecommunication, Inha University, Inha, Korea.

K. Sarabandi is with the Radiation Laboratory, Department of Electrical Engineering and Computer Science, The University of Michigan, Ann Arbor, 48109-

2122 MI USA (e-mail: saraband@eecs.umich.edu).

Digital Object Identifier 10.1109/TAP.2005.848463

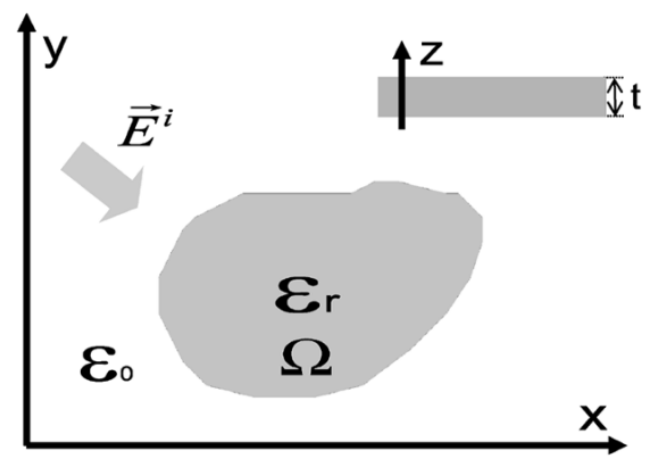

(a)

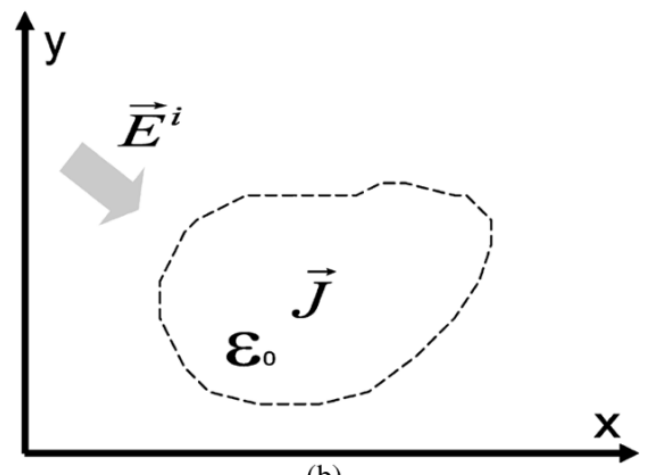

(b)

Fig. 1.Very thin planar dielectric disk with thickness $t$, located in $x y$ plane of (a) a coordinate system and (b) its equivalent volumetric polarization current.

rate results independent of the thickness but for disks of large dimensions compared to the wavelength. This solution fails for near edge-on incidence. Therefore at intermediate frequencies and for near-grazing incidence at high frequencies, prediction of scattering from these structures using existing methods becomes erroneous. On the other hand for large scatterers, numerical methods like MoM become numerically inefficient. Hence an accurate analytical formulation that can cover these gaps is of high interest.

The integral equation based on volumetric equivalent current has been well-formulated and used intensively [6]. As pointed out in [7] the volumetric integral equation (VIE) is not usually used in MoM algorithms since the required memory is much higher than that needed for the surface integral formulations. However, VIE has been used to obtain approximate solutions for the polarization currents inside dielectric scatterers based on the well-known Neumann(Born) series approach [8], [9], and rough surface problems [10], [11] using the small perturbation method. In this paper, VIE is used to formulate scattering from thin dielectric objects where approximations pertaining to the small thickness of the objects and Fourier trans- 
form are employed. The proposed solution is shown to be valid for a wide range of dielectric constants, and incidence angles. In Section II, the general formulation for polarization current inside an arbitrarily shaped 3-D planar dielectric scatterer is presented. Then in the successive sections, the general solution is applied to a number of canonical objects for model verification. Throughout this paper, a time convention of $e^{-i \omega t}$ is assumed and suppressed.

\section{FORMULATION}

Fig. 1(a) shows the problem geometry in which a very thin dielectric scatterer is located in $x-y$ plane of a cartesian coordinate system. The scatterer is assumed homogeneous with a relative permittivity $\varepsilon_{r}$ and arbitrary shape having a constant thickness $t \ll \lambda$. Using the notation of polarization current, the scatterer can be replaced with a volumetric current distribution of $\vec{J}(x, y, z)=i k_{0} Y_{0}\left(\varepsilon_{r}-1\right) \vec{E}^{t}$ embedded in the host medium with permittivity $\varepsilon_{0}$. Here $\vec{E}^{t}=\vec{E}^{i}+\vec{E}^{s}$ is the total electric field inside the scatterer. It can easily be shown that $\vec{J}$ must satisfy the following Fredholm integral equation:

$$
\vec{J}(\vec{r})-k_{0}^{2}\left(\varepsilon_{r}-1\right) \int_{v^{\prime}} \overline{\bar{G}}\left(\vec{r}, \vec{r}^{\prime}\right) \cdot \vec{J}\left(\vec{r}^{\prime}\right) d v^{\prime}=-i k_{0} Y_{0}\left(\varepsilon_{r}-1\right) \vec{E}^{i}(\vec{r})
$$

where $k_{0}$ is the free space wave number, $Y_{0}=1 / Z_{0}$ is the free space characteristic admittance, $\vec{E}^{i}$ is the incident wave, and $\overline{\bar{G}}$ is the free-space dyadic Green's function [8]. The integral, $\int_{v^{\prime}}$, in (1) is performed over the whole volume of the scatterer. To arrive at an approximate form for the intended solution, we begin with the spectral representation of the dyadic Green's function given by

$$
\begin{aligned}
\overline{\bar{G}}\left(\vec{r}, \vec{r}^{\prime}\right)=-\hat{z} \hat{z} \frac{\delta\left(\vec{r}-\vec{r}^{\prime}\right)}{k_{0}^{2}}+\frac{i}{8 \pi^{2}} \int_{-\infty}^{\infty} \int^{2} k \frac{e^{i\left[k_{x}\left(x-x^{\prime}\right)+k_{y}\left(y-y^{\prime}\right)\right]}}{k_{z}} \\
\cdot \begin{cases}{\left[\hat{h} \hat{h}+\hat{v}_{+} \hat{v}_{+}\right] e^{i k_{z}\left(z-z^{\prime}\right)}} & \text { if } z>z^{\prime} \\
{\left[\hat{h} \hat{h}+\hat{v}_{-} \hat{v}_{-}\right] e^{-i k_{z}\left(z-z^{\prime}\right)}} & \text { if } z<z^{\prime}\end{cases}
\end{aligned}
$$

where $k_{x}^{2}+k_{y}^{2}+k_{z}^{2}=k_{0}^{2}$, and $\hat{h}, \hat{v}_{+}$, and $\hat{v}_{-}$are defined as

$$
\hat{h}=\frac{\hat{k}^{i} \times \hat{z}}{\left|\hat{k}^{i} \times \hat{z}\right|}, \quad \hat{v}_{+}=\hat{h} \times \hat{k}^{i}, \quad \hat{v}_{-}=\hat{h} \times \hat{k}^{r} .
$$

Here, $\hat{k}^{i}=\left(1 / k_{0}\right)\left(k_{x}^{i} \hat{x}+k_{y}^{i} \hat{y}+k_{z}^{i} \hat{z}\right)$, and $\hat{k}^{r}=\left(1 / k_{0}\right)\left(k_{x}^{i} \hat{x}+\right.$ $\left.k_{y}^{i} \hat{y}-k_{z}^{i} \hat{z}\right)$. It should be pointed out that position vector $\vec{r}$ in (1) belongs to a set of points inside the scatterer as indicated by the term $\left(\varepsilon_{r}-1\right)$ and the current $\vec{J}$ itself. By substituting (2) in (1) and then evaluating the delta function term in (2) analytically, the VIE can be expressed in a more compact form given by

$$
\vec{J}-\overline{\bar{A}} k_{0}^{2}\left(\varepsilon_{r}-1\right) \int_{v^{\prime}} \overline{\bar{G}}_{r} \cdot \vec{J} d v^{\prime}=\vec{J}_{\mathrm{RG}}
$$

where $\overline{\bar{G}}_{r}$ is the integral part in (2), and $\vec{J}_{\mathrm{RG}}$ is the well-known Rayleigh-Gans current [5] given by $\vec{J}_{\mathrm{RG}}=-i k_{0} Y_{0}\left(\varepsilon_{r}-1\right) \overline{\bar{A}} \vec{E}^{i}$ and $\bar{A}$ is a diagonal matrix defined by $\bar{A}=\hat{x} \hat{x}+\hat{y} \hat{y}+\left(1 / \varepsilon_{r}\right) \hat{z} \hat{z}$. Basically the Rayleigh-Gans solution can be interpreted as the zeroth order solution of the modified VIE (3).

As mentioned, $\vec{J}$ and $\vec{J}_{\mathrm{RG}}$ are nonzero only inside the scatterer (see Fig. 1), i.e.

$$
\vec{J}_{\mathrm{RG}}, \vec{J}(x, y, z) \begin{cases}\neq 0 & (x, y, z) \in \Omega \\ =0 & (x, y, z) \notin \Omega\end{cases}
$$

Therefore, when taking Fourier transform, the infinite integral range can be truncated over a finite range of $\Omega$ as

$$
\iint_{-\infty}^{\infty} \vec{J} e^{i\left(k_{x} x+k_{y} y+k_{z} z\right)} d v=\iiint_{\Omega} \vec{J} e^{i\left(k_{x} x+k_{y} y+k_{z} z\right)} d v .
$$

By invoking the thin dielectric approximation, the polarization current can be assumed a constant with respect to $z$-axis, i.e., $\vec{J}(x, y, z) \approx \vec{J}(x, y, 0)$. Hence the integral in (3) with respect to $z$ can be evaluated using the mid-point approximation and we have

$$
\begin{aligned}
\vec{I} & =\int_{v^{\prime}} \overline{\bar{G}}_{r}\left(\vec{r} ; \vec{r}^{\prime}\right) \cdot \vec{J}\left(\vec{r}^{\prime}\right) d v^{\prime} \\
& \approx t \int_{s^{\prime}} \overline{\bar{G}}_{r}\left(x, y, 0 ; x^{\prime}, y^{\prime}, 0\right) \cdot \vec{J}\left(x^{\prime}, y^{\prime}, 0\right) d s^{\prime}
\end{aligned}
$$

Also, according to thin dielectric assumption, $(\partial / \partial z) J_{x}=$ $(\partial / \partial z) J_{y}=0$, and therefore

$$
\vec{I} \approx \frac{t}{8 \pi^{2}} \int_{-\infty}^{\infty} \int^{2} d^{2} \frac{\overline{\bar{A}} \cdot \overline{\bar{L}}}{k_{z}} \int_{s^{\prime}} d s^{\prime} \vec{J}\left(x^{\prime}, y^{\prime}\right) e^{i\left[k_{x}\left(x-x^{\prime}\right)+k_{y}\left(y-y^{\prime}\right)\right]}
$$

where $\overline{\bar{L}}$ is given by

$$
\overline{\bar{L}}=\frac{1}{k_{0}^{2}}\left(\begin{array}{ccc}
k_{0}^{2}-k_{x}^{2} & -k_{x} k_{y} & 0 \\
-k_{x} k_{y} & k_{0}^{2}-k_{y}^{2} & 0 \\
0 & 0 & k_{0}^{2}-k_{z}^{2}
\end{array}\right)
$$

Now the original integral equation given by (3) can be written as

$$
\begin{aligned}
& \vec{J}-t \frac{k_{0}^{2}}{8 \pi^{2}}\left(\varepsilon_{r}-1\right) \iint_{-\infty}^{\infty} d^{2} k \frac{\overline{\bar{A}} \cdot \overline{\bar{L}}}{k_{z}} \times \\
& \int_{s^{\prime}} d s^{\prime} \vec{J}\left(x^{\prime}, y^{\prime}\right) e^{i\left[k_{x}\left(x-x^{\prime}\right)+k_{y}\left(y-y^{\prime}\right)\right]}=\vec{J}_{\mathrm{RG}}
\end{aligned}
$$

Using (4), the embedded integral in (6) can be interpreted as a convolution integral. Taking the Fourier transform of both sides 
and using $\left(\varepsilon_{r}-1\right)$ is a constant function of position, (6) can be solved analytically. Basically it can be shown that

$$
\tilde{J}=\overline{\bar{B}}^{-1} \tilde{J}_{\mathrm{RG}}
$$

where $\tilde{J}$ and $\tilde{J}_{\mathrm{RG}}$ are the Fourier transform of $\vec{J}$ and $\vec{J}_{\mathrm{RG}}$, respectively. Also $\overline{\bar{B}}^{-1}=\left[\overline{\bar{I}}-\alpha k_{0}^{2}\left(\overline{\bar{A}} \cdot \overline{\bar{L}} / k_{z}\right)\right]^{-1}, \overline{\bar{I}}$ is an unit dyadic, and $\alpha=(i / 2) t\left(\varepsilon_{r}-1\right)$. Explicitly $\overline{\bar{B}}^{-1}$ can be expressed as

$\overline{\bar{B}}^{-1}=\left(\begin{array}{ccc}\frac{1}{D}\left[k_{z}-\alpha\left(k_{0}^{2}-k_{y}^{2}\right)\right] & -\frac{\alpha}{D} k_{x} k_{y} & 0 \\ -\frac{\alpha}{D} k_{x} k_{y} & \frac{1}{D}\left[k_{z}-\alpha\left(k_{0}^{2}-k_{x}^{2}\right)\right] & 0 \\ 0 & 0 & \frac{\varepsilon_{r} k_{z}}{\varepsilon_{r} k_{z}-\alpha k_{\rho}^{2}}\end{array}\right)$

where $D=k_{z}\left(1+\alpha^{2} k_{0}^{2}\right)-\alpha\left(k_{0}^{2}+k_{z}^{2}\right)=\left(1-\alpha k_{z}\right)\left(k_{z}-\alpha k_{0}^{2}\right)$, and $k_{\rho}^{2}=k_{x}^{2}+k_{y}^{2}$. By taking the inverse Fourier transform, a closed-form expression of $\vec{J}$ can be obtained and is given by

$$
\begin{aligned}
\vec{J}(x, y) \approx \frac{1}{4 \pi^{2}} \int_{-\infty}^{\infty} \int^{2} k \overline{\bar{B}}^{-1} e^{i\left(k_{x} x+k_{y} y\right)} & \\
& \times \int_{s^{\prime}} d s^{\prime} \vec{J}_{\mathrm{RG}} e^{-i\left(k_{x} x^{\prime}+k_{y} y^{\prime}\right)} .
\end{aligned}
$$

In reality, the quantity $\left(\varepsilon_{r}-1\right)$ is not a constant function of position in the entire domain. Hence in the Fourier transform operation, we should have included a convolution of the object shape function with the right-hand side of (6). Ignoring the convolution process can be justified at both low and high frequency regimes. Since the Fredholm integral equation (6) is of second kind, the contribution from the integral when the extent of the scatterer is small compared with the wavelength can be ignored. This is rather clear in the context of the discretized version of the integral equation (method of moment approach). At low frequencies the so-called impedance matrix has a small size and its diagonal elements are close to unity and much larger than those contributed by the integral term. That is why ignoring the entire integral, and thus the associated convolution process, yield the familiar traditional solution for $\vec{J} \approx \vec{J}_{\mathrm{RG}}$ at low frequencies. At high frequencies where the Fourier transform of the shape function approaches a delta function, the convolution process does not change the results. Hence the formal solution presented by (8) is valid at both low and high frequencies. In between so long as the product $\left(\varepsilon_{r}-1\right) \vec{I}$ is a relatively small quantity, the errors caused by ignoring the convolution process should be small. This will be shown in the following sections where the results obtained from (8) are compared with those obtained from numerical and exact solutions.

The scattered field in the far-field region can also be obtained easily from (8). Considering a plane wave incidence, $\vec{E}^{i}=\vec{e}_{i} e^{i\left(k_{x}^{i} x+k_{y}^{i} y+k_{z}^{i} z\right)}$, the surface integral term in (8) can be rewritten as

$$
\begin{aligned}
\int_{s^{\prime}} \vec{J}_{\mathrm{RG}} e^{-i\left(k_{x} x^{\prime}+k_{y} y^{\prime}\right)} d s^{\prime}= & -i k_{0} Y_{0}\left(\varepsilon_{r}-1\right) \overline{\bar{A}} \\
& \cdot \vec{e}_{i} \int_{s^{\prime}} e^{i\left[\left(k_{x}^{i}-k_{x}\right) x^{\prime}+\left(k_{y}^{i}-k_{y}\right) y^{\prime}\right]} d s^{\prime}
\end{aligned}
$$

and field can be calculated as

$$
\begin{aligned}
\vec{E} \sim & i\left(\varepsilon_{r}-1\right) \overline{\bar{A}} \cdot \vec{e}_{i} \frac{k_{0}^{3} Y_{0}}{16 \pi^{3}} \frac{e^{k_{0} r}}{r} \hat{r} \times \hat{r} \times \int_{-\infty}^{\infty} \int^{2} k \overline{\bar{B}}^{-1} \\
& \times \int_{s^{\prime}} d s^{\prime} e^{i\left[\left(k_{x}^{i}-k_{x}\right) x^{\prime}+\left(k_{y}^{i}-k_{y}\right) y^{\prime}\right]} \\
& \times \int_{s^{\prime \prime}} d s^{\prime \prime} e^{-i\left[\left(k_{x}^{s}-k_{x}\right) x^{\prime \prime}+\left(k_{y}^{s}-k_{y}\right) y^{\prime \prime}\right] .}
\end{aligned}
$$

Here, $\hat{k}^{s}=\left(1 / k_{0}\right)\left(k_{x}^{s} \hat{x}+k_{y}^{s} \hat{y}+k_{z}^{s} \hat{z}\right)$ is a unit vector along the direction of observation point. For canonical geometries these embedded integrals can be calculated analytically as will be shown later. It is also worth mentioning that the convergence of the spectral integrals improves at higher frequencies since the integrand decays faster.

\section{INFINITE DIELECTRIC SLAB}

The first example chosen for verifying results is an infinite dielectric slab for which an exact solution is known [5]. For this example, the approximate solution given by (8) is exact. For simplicity, a plane wave excitation is assumed, but the results can be easily extended to any arbitrary excitation using superposition principle. Since the extent of the scatterer is infinite, all integrals in (8) can be evaluated analytically in terms of a diagonal polarizability tensor like $\vec{J}=-i t k_{0} Y_{0}\left(\varepsilon_{r}-1\right)\left[P_{x x} E_{x}^{i} \hat{x}+\right.$ $\left.P_{y y} E_{y}^{i} \hat{y}+P_{z z} E_{z}^{i} \hat{z}\right]$. After some algebraic manipulations, the elements of the polarizability tensor can be obtained as

$$
\begin{aligned}
P_{x x}= & P_{y y}=\frac{k_{z}^{i}}{k_{z}^{i}-\alpha k_{0}^{2}}, \quad P_{z z}=0 \\
& \text { for } h-\text { pol. incidence } \\
P_{x x}= & P_{y y}=\frac{1}{1-\alpha k_{z}^{i}}, \quad P_{z z}=\frac{k_{z}^{i}}{\varepsilon_{r} k_{z}^{i}-\alpha k_{\rho}^{i^{2}}} \\
& \text { for } v-\text { pol. incidence }
\end{aligned}
$$

where $k_{\rho}^{i^{2}}=k_{x}^{i^{2}}+k_{y}^{i^{2}}$. The exact solutions as given in [5] can be slightly modified to show

$$
\begin{aligned}
& P_{x x}=P_{y y}=\frac{2 k_{z}^{i}}{\triangle_{1}}, \quad P_{z z}=0 \quad \text { for } h-\text { pol. incidence, } \\
& P_{x x}=P_{y y}=\frac{2 k_{z}^{\prime}}{\triangle_{2}}, \quad P_{z z}=\frac{2 k_{z}^{i}}{\triangle_{3}} \quad \text { for } v-\text { pol. incidence }
\end{aligned}
$$

where $\Delta_{1}=\left(k_{z}^{i}+k_{z}^{\prime}\right) e^{-i\left(k_{z}^{\prime}-k_{z}^{i}\right) t / 2}+\left(k_{z}^{i}-k_{z}^{\prime}\right) e^{i\left(k_{z}^{\prime}+k_{z}^{i}\right) t / 2}$, $\Delta_{2}=\left(\varepsilon_{r} k_{z}^{i}+k_{z}^{\prime}\right) e^{-i\left(k_{z}^{\prime}-k_{z}^{i}\right) t / 2}-\left(\varepsilon_{r} k_{z}^{i}-k_{z}^{\prime}\right) e^{i\left(k_{z}^{\prime}+k_{z}^{i}\right) t / 2}$, $\Delta_{3}=\left(\varepsilon_{r} k_{z}^{i}+k_{z}^{\prime}\right) e^{-i\left(k_{z}^{\prime}-k_{z}^{i}\right) t / 2}+\left(\varepsilon_{r} k_{z}^{i}-k_{z}^{\prime}\right) e^{i\left(k_{z}^{\prime}+k_{z}^{i}\right) t / 2}$, and $k_{z}^{\prime}=\sqrt{\varepsilon_{r} k_{0}^{2}-k_{\rho}^{i^{2}}}$. Using the small thickness approximation, the exponential terms can be approximated by their first-order Taylor series expansion, i.e., $e^{i\left(k_{z}^{\prime}+k_{z}^{i}\right) t / 2} \approx 1+i(t / 2)\left(k_{z}^{\prime}+k_{z}^{i}\right)$. Using this approximation

$$
\begin{aligned}
& \triangle_{1} \approx 2 k_{z}^{\prime}+i t\left(k_{z}^{i^{2}}-{k_{z}^{\prime}}^{2}\right)=2 k_{z}^{i}-2 \alpha k_{0}^{2} \\
& \triangle_{2} \approx 2 k_{z}^{\prime}-i t k_{z}^{\prime} k_{z}^{i}\left(\varepsilon_{r}-1\right)=2 k_{z}^{\prime}-2 \alpha k_{z}^{\prime} k_{z}^{i} \\
& \triangle_{3} \approx 2 \varepsilon_{r} k_{z}^{i}-i t\left(\varepsilon_{r}-1\right) k_{\rho}^{i^{2}}=2 \varepsilon_{r} k_{z}^{i}-2 \alpha k_{\rho}^{i^{2}}
\end{aligned}
$$

which obviously produce results identical to (9) and (10). 


\section{Dielectric StRIP AND Half-Plane}

The next example considered here is the problem of scattering from a thin dielectric strip whose geometry is shown in Fig. 2(a). For a plane wave incidence, the polarization current (8) can be reduced to a single integral given by

$$
\begin{aligned}
& \vec{J} \approx-i k_{0} Y_{0}\left(\varepsilon_{r}-1\right) \frac{w}{2 \pi} \int_{-\infty}^{\infty} d k_{x} \overline{\bar{B}}_{1}^{-1} \overline{\bar{A}} \\
& \cdot \vec{e}_{i} \operatorname{sinc}\left[\frac{\left(k_{x}^{i}-k_{x}\right)}{2} w\right] e^{i\left(k_{x} x+k_{y}^{i} y\right)}
\end{aligned}
$$

where $\overline{\bar{B}}_{1}^{-1}$ is the same as $\overline{\bar{B}}^{-1}$ with $k_{y}=k_{y}^{i}$. The corresponding electric field in the far-field region can be easily formulated as

$$
\begin{array}{r}
\vec{E} \sim i t\left(\varepsilon_{r}-1\right) \frac{\left(k_{0} w\right)^{2}}{8 \pi} \sqrt{\frac{2}{\pi k_{0} \rho}} e^{i\left(k_{0} \rho-\frac{\pi}{4}\right)} \cdot \hat{r} \times \hat{r} \times \int_{-\infty}^{\infty} d k_{x} \overline{\bar{B}}_{1}^{-1} \overline{\bar{A}} \\
\cdot \vec{e}_{i} \operatorname{sinc}\left[\frac{k_{x}^{i}-k_{x}}{2} w\right] \text { sinc }\left[\frac{k_{x}-k_{x}^{s}}{2} w\right] . \quad \text { (12) }
\end{array}
$$

Unfortunately the integral can't be evaluated analytically and must be calculated numerically. To examine the validity of (12), examples are chosen from [12]. Fig. 2 shows comparisons of normalized radar echo width of a strip with thickness $0.025 \lambda_{0}$ and $\varepsilon_{r}=4+i 0.4$ as a function of $w / \lambda_{0}$, which are calculated from (12) and MoM in forward and backward directions for an edge-on TM polarized incident wave. Edge-on incidence is chosen as it is the most complicated scenario for the scattering problem. Fig. 2 shows that the approximate formulation (12) provides excellent results over the entire comparison region. In this calculation, the width of the strip is set to be $5 \lambda_{0}$ and $50 \lambda_{0}$.

Next, backscattering from a thin dielectric half-plane is considered. For this structure, exact and approximate solutions are known [1], [13]. The problem geometry consisting of a dielectric half-plane located along the negative $x$-axis is shown in Fig. 3. For this geometry, (8) is reduced to

$$
\vec{J}=\frac{1}{2} \vec{J}_{\mathrm{inf}}-i k_{0} Y_{0}\left(\varepsilon_{r}-1\right) \frac{i}{2 \pi} \int_{-\infty}^{\infty} d k_{x} \overline{\bar{B}}_{1}^{-1} \overline{\bar{A}} \cdot \vec{e}_{i} \frac{e^{i\left(k_{x} x+k_{y}^{i} y\right)}}{k_{x}-k_{x}^{i}}
$$

where $\vec{J}_{\text {inf }}$ is the current inside an infinite slab (PO current). The integral in (13) contains a singularity at $k_{x}=k_{x}^{i}$ whose contribution to the integral, using the residue theorem, provides the complete PO current. If we focus on just edge-on incidence case, this PO current always becomes zero. Considering a TM polarized wave having an electric field along $\hat{y}$ and $k_{y}^{i}=0$, in the far-field region, the electric field can be written as $\vec{E} \sim \hat{y} \sqrt{2 / \pi k_{0} \rho} e^{i\left(k_{0} \rho-\pi / 4\right)} P_{e}\left(\theta_{i}, \theta_{s}\right)$, where $P_{e}\left(\theta_{i}, \theta_{s}\right)$ is known as the far-field amplitude. $P_{e}\left(\theta_{i}, \theta_{s}\right)$ can be computed for the backscattering direction from

$$
P_{e}\left(\frac{\pi}{2}, \frac{\pi}{2}\right)=\frac{k_{0}}{4 \pi \eta} \int_{-\infty}^{\infty} d k_{x} \frac{1}{k_{z}\left(k_{z}-\alpha k_{0}^{2}\right)}
$$

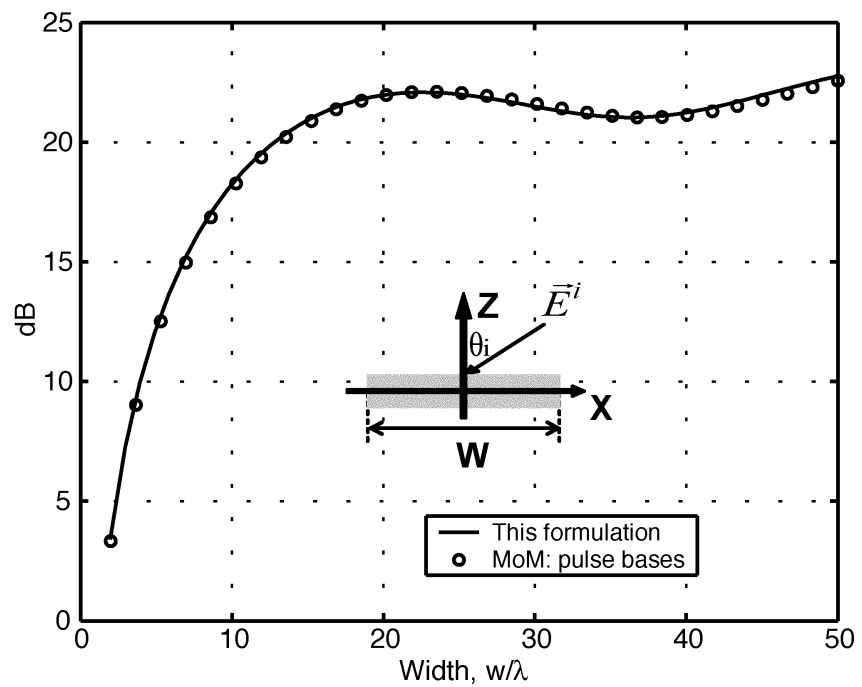

(a)

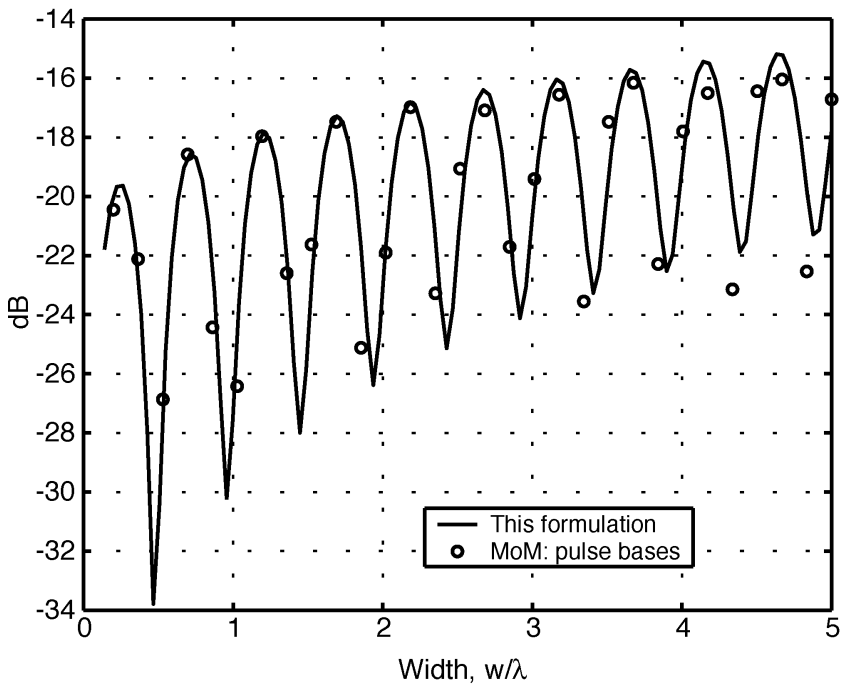

(b)

Fig. 2. Plot of radar cross sections of a dielectric strip normalized by wavelength $(\sigma / \lambda)$ as a function of width for edge-on TM wave incidence case Thickness of the strip is $0.025 \lambda_{0}$, and the dielectric constant is $4+i 0.4$. (a) Forward scattering and (b) backscattering.

where $\alpha k_{0}=-(1 / \eta)$, and $\eta=2 R / Z_{0}$ is the normalized surface impedance, and $R=i Z_{0} / k_{0} t\left(\varepsilon_{r}-1\right)$. The integral in the above equation can be evaluated analytically as

$$
\begin{aligned}
P_{e}\left(\frac{\pi}{2}, \frac{\pi}{2}\right)= & -\frac{1}{\pi \sqrt{1-\eta^{2}}} \\
& \times\left[\tan ^{-1} \frac{\eta-1}{\sqrt{1-\eta^{2}}}-i \tan h^{-1} \frac{\sqrt{\eta^{2}-1}}{\eta-i}\right] \\
= & -\frac{i}{\pi \sqrt{1-\eta^{2}}} \tan h^{-1} \sqrt{\frac{1-\eta}{1+\eta}}
\end{aligned}
$$

In a special case $\eta=1$, (14) may be computed analytically as $-(i / 2 \pi)$. An exact solution for a resistive half-plane [13] provides $P_{e}(\pi / 2, \pi / 2)=-(i / 4) \cdot 0.7894^{2}=-i 0.1558$, which is in agreement with the new result within $2 \%$ error. To further validate the new formulation for arbitrary values of $\eta$, a known asymptotic solution for a resistive half-plane problem is also 


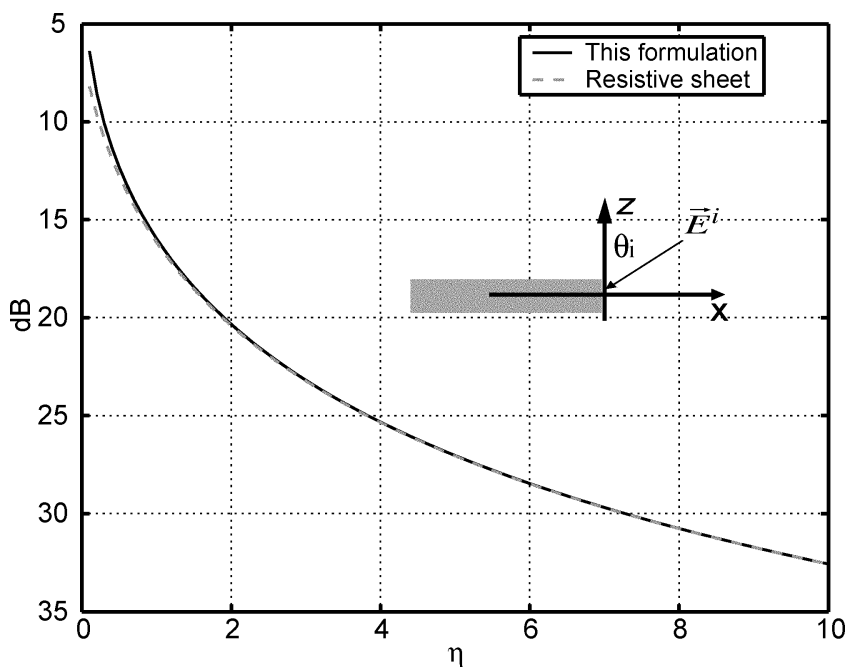

Fig. 3. Backscattering amplitude $\left(P_{e}\right)$ from a very thin dielectric half-plane as a function of $\eta$ (real) for the edge-on TM wave incidence case. Comparison is shown between the new formulation and results obtained from an independent method based on resistive sheet.

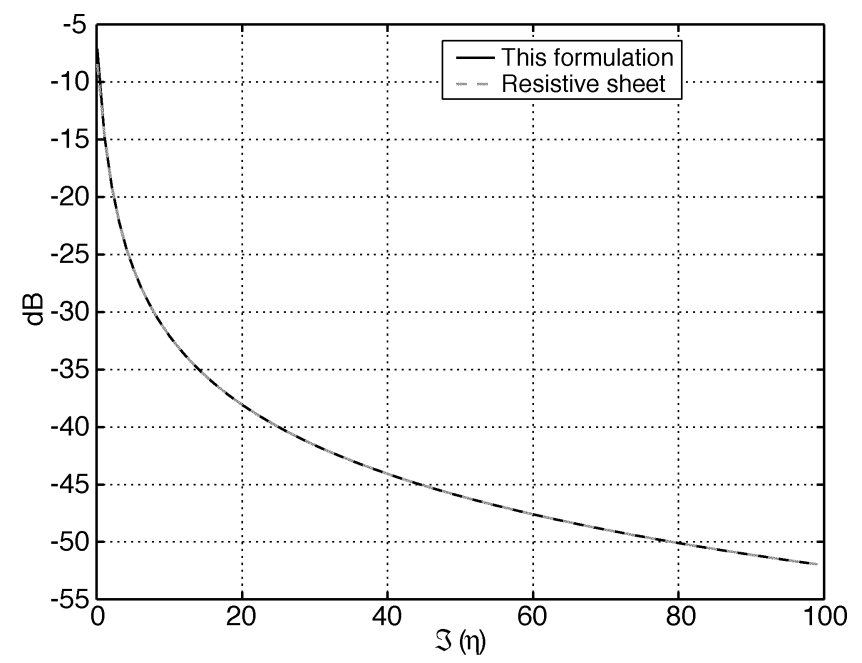

(a)

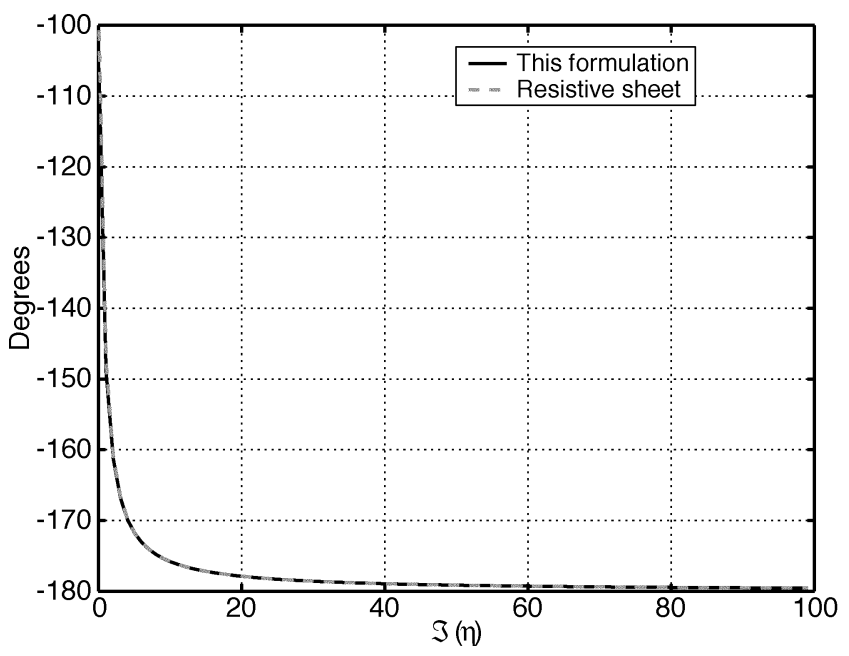

(b)

Fig. 4. (a) Magnitude and (b) phase of backscattering amplitude $\left(P_{e}\right)$ from a dielectric half-plane as a function of imaginary part of $\eta$ for the edge-on TM wave incidence. In this case the real part of $\eta$ is kept constant at 0.1 .

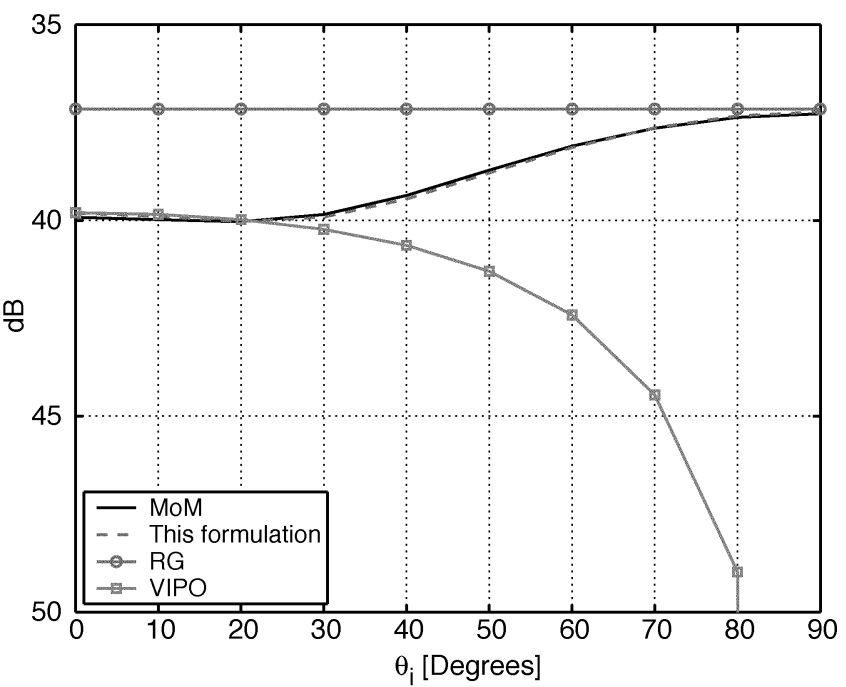

(a)

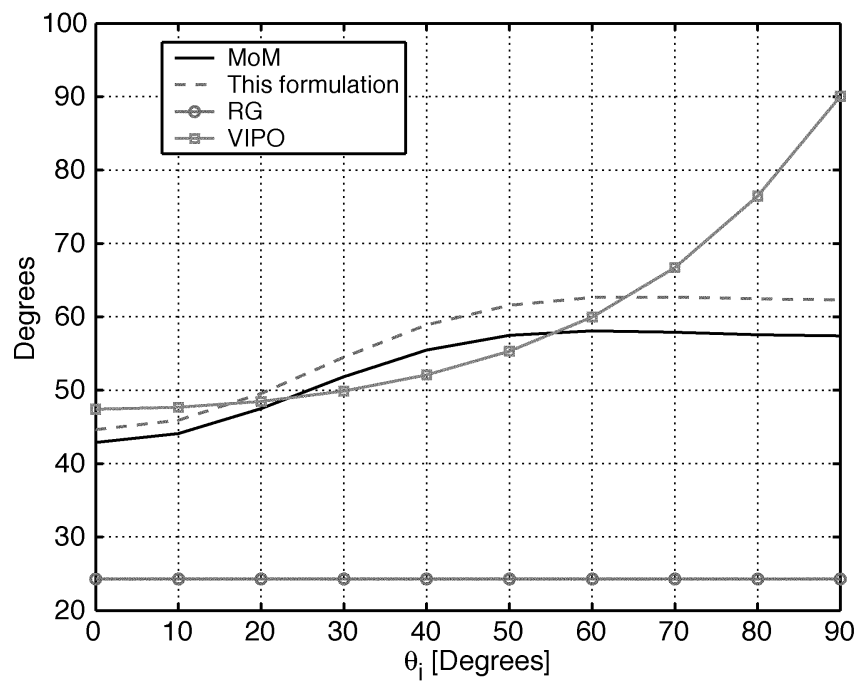

(b)

Fig. 5. (a) Magnitude and (b) phase of forward scattering for hh polarization $\left(S_{h h}\right)$ from a circular disk with a diameter of $3 \mathrm{~cm}$, thickness of $0.2 \mathrm{~mm}$, and dielectric constant of $26.6+i 11.56$ at $10 \mathrm{GHz}$. The results based on four methods are shown: the new formulation, MoM, VIPO, and Rayleigh-Gans.

used [13], which provides

$$
P_{e}\left(\frac{\pi}{2}, \frac{\pi}{2}\right)=-i \frac{\eta}{16} Z J(0)
$$

$Z J(0)$ is represented by a contour integral including Maliuzhinets half-plane function whose definition and approximations can be found in [1]. Fig. 3 shows a comparison of the results calculated by (14) and (15) as a function of $\eta$ (real in this case). An excellent agreement is observed. However, it is shown that the discrepancy is increased with decreasing $\eta$ which corresponds to increasing $\varepsilon_{r}$. As mentioned before, it is expected for the accuracy of the proposed formulation to degrade for large values of $t\left(\varepsilon_{r}-1\right) / \lambda$. From Fig. 3 it is founded that when $\eta$ is larger than 0.5 , the difference between the two solutions is less than $0.5 \mathrm{~dB}$. For a perfect electrical conductor (PEC) case, $\eta=0$, this formulation is no longer valid because the Fredholm integral equation reduces to the first kind. Fig. 4 is a plot of $P_{e}$ as a function of the imaginary part of $\eta$ and a fixed real part of 0.1 . As seen from this figure the new formulation agrees very well with the asymptotic results. Hence (14) can provide a very 


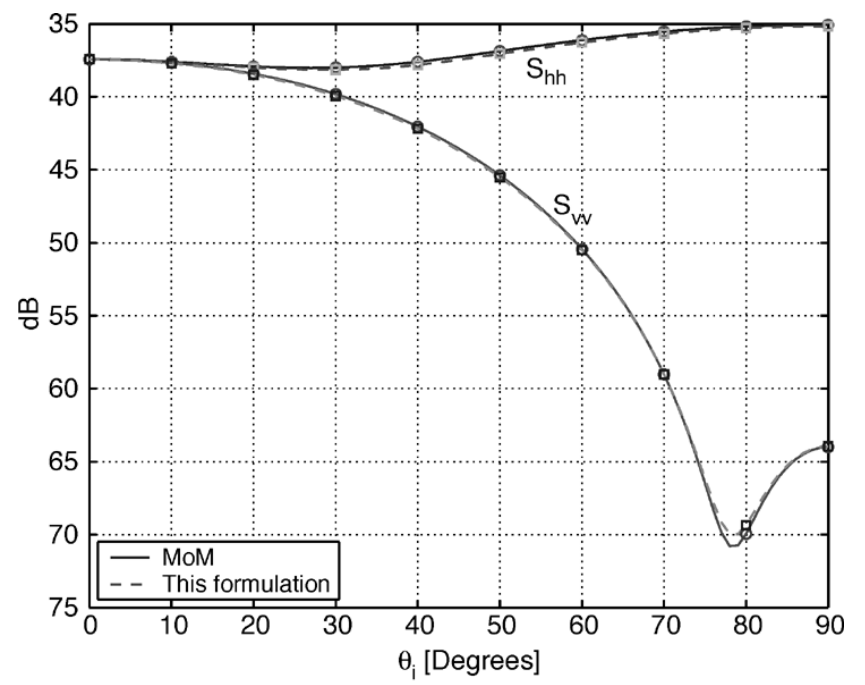

(a)

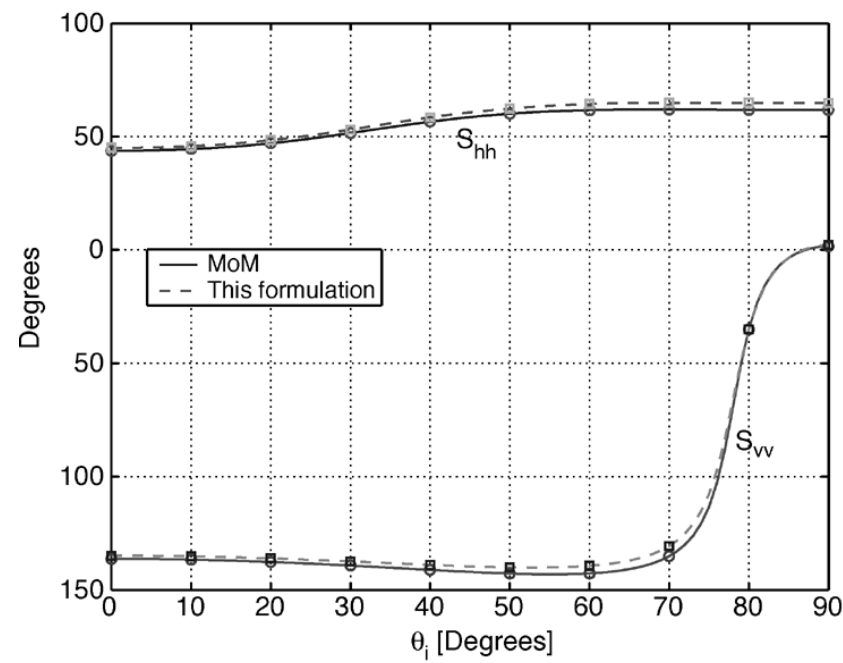

(b)

Fig. 6. (a) Magnitude and (b) phase of backscattering amplitudes $\left(S_{h h}\right.$, and $S_{v v}$ ) of a square disk with $w_{x}=w_{y}=3 \mathrm{~cm}$, thickness of $0.2 \mathrm{~mm}$, and dielectric constant of $26.6+i 11.56$ at $10 \mathrm{GHz}$. The results based on two methods are shown: the new formulation and MoM.

good approximation for the Maliuzhinets half-plane function for moderate to large value of $|\eta|$.

\section{DIELECTRIC DISK}

In this section scattering by a very thin dielectric disk is examined. For this geometry, the formulations derived in Section II can be directly used. First, scattering from an elliptical disk with major and minor axes of $a$ and $b$ is investigated. Choosing a coordinate system with $\mathrm{x}$ along the major axis and $\mathrm{y}$ along the minor axis of the ellipse, the surface integral of (8) can be evaluated in a closed-form and is given by [14]

$$
\begin{aligned}
\left.\iint_{s^{\prime}} e^{i\left[\left(k_{x}-k_{z}^{i}\right) x^{\prime}\right.}+\left(k_{y}-k_{y}^{i}\right) y^{\prime}\right] d s^{\prime} \\
=\frac{2 A J_{1}\left(\sqrt{a^{2}\left(k_{x}-k_{x}^{i}\right)^{2}+b^{2}\left(k_{y}-k_{y}^{i}\right)^{2}}\right)}{\sqrt{a^{2}\left(k_{x}-k_{x}^{i}\right)^{2}+b^{2}\left(k_{y}-k_{y}^{i}\right)^{2}}}
\end{aligned}
$$

where $A=\pi a b$ is the area of the ellipse and $J_{1}(\cdot)$ is the Bessel function of the first kind of first order. Since this structure is

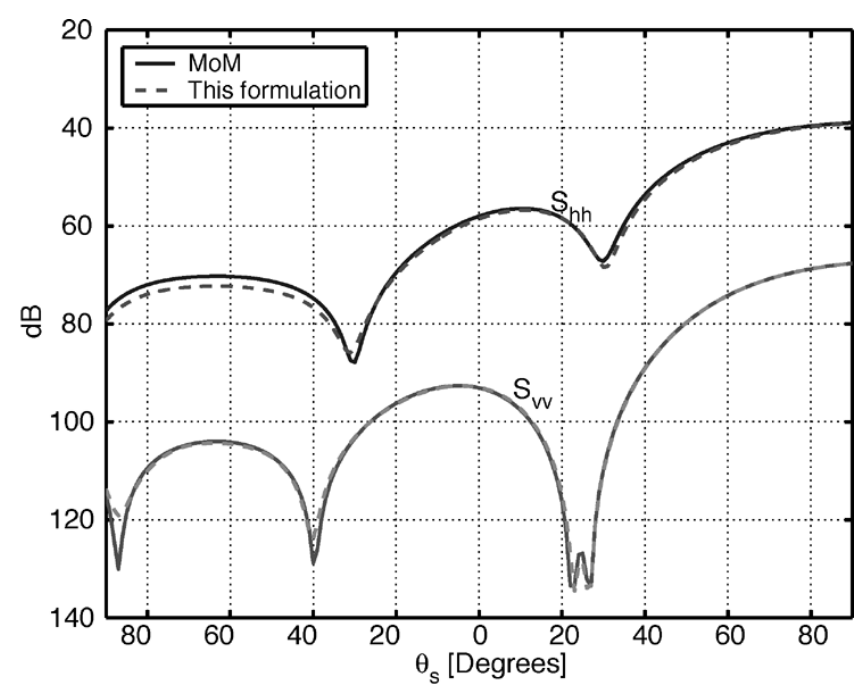

(a)

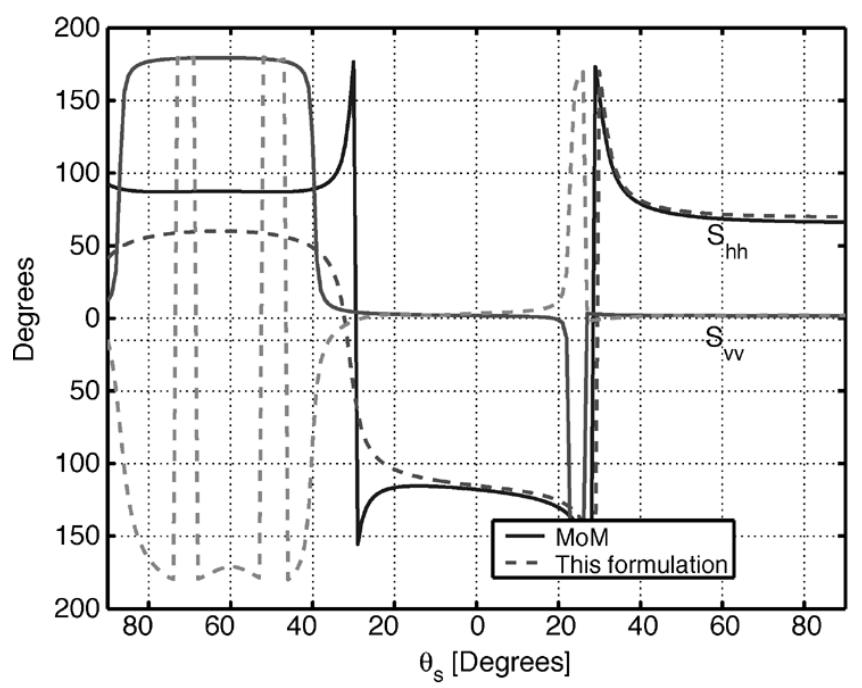

(b)

Fig. 7. (a) Magnitude and (b) phase of bistatic scattering of $S_{h h}$ and $S_{v v}$ from a square disk with $w_{x}=w_{y}=3 \mathrm{~cm}$, thickness of $0.2 \mathrm{~mm}$, and dielectric constant of $26.6+i 11.56$ at $10 \mathrm{GHz}$. The results based on two methods are shown: the new formulation and MoM. $\theta_{i}$ is fixed to be $90^{\circ}$, and $\theta_{s}$ moves $-90^{\circ}$ to $90^{\circ}$ with $\phi_{i}=180^{\circ}$, and $\phi_{s}=45^{\circ}$.

widely used to model vegetation leaves, for the examples considered here a typical permittivity value of a leaf at $10 \mathrm{GHz}$ $\left(\varepsilon_{r}=26.6+i 11.56\right)$ is used. Forward scattering from a circular disk with a diameter of $3 \mathrm{~cm}$, and a thickness of $0.2 \mathrm{~mm}$ is considered. Fig. 5 shows a plot of the scattering of the dielectric disk in forward direction as a function of incidence angles. In this figure two approximate formulations such as VIPO (high frequencies) and Rayleigh-Gans (low frequencies) as well as MoM results are shown. It is shown that the new formulation can reproduce the MoM results very accurately over the entire comparison region where low and high frequency results are only valid at high and low incidence angles only.

Another geometry for which the surface integral in (8) can be carried out analytically is a rectangle. For this shape

$$
\begin{aligned}
\iint_{s^{\prime}} e^{i\left[\left(k_{x}-k_{x}^{i}\right) x^{\prime}+\left(k_{y}-k_{y}^{i}\right) y^{\prime}\right]} d s^{\prime} \\
=w_{x} w_{y} \operatorname{sinc}\left[\frac{k_{x}^{i}-k_{x}}{2} w_{x}\right] \operatorname{sinc}\left[\frac{k_{y}^{i}-k_{y}}{2} w_{y}\right]
\end{aligned}
$$


where $w_{x}$ and $w_{y}$ are the width and length of the rectangular disk, respectively. Fig. 6 shows a plot of radar backscatter from a square leaf with $w_{x}=w_{y}=3 \mathrm{~cm}$ and thickness of $t=0.2 \mathrm{~mm}$ as a function of incidence angles at $10 \mathrm{GHz}$. The difference between the MoM and new formulation is less than $0.2 \mathrm{~dB}$ in magnitude and $4.5^{\circ}$ in phase. Also the bistatic scattering radar cross section from the square disk is calculated for an incident wave propagating along the negative $x$-axis (edge-on incidence). The observation point $\left(\theta_{s}\right)$ is varied from $-90^{\circ}$ to $90^{\circ}$ along the diagonal direction of the disk $\left(\phi_{s}=45^{\circ}\right)$. Fig. 7 shows a plot of $S_{h h}$, and $S_{v v}$ as a function of $\theta_{s}$. Some small discrepancy is observed in the backward direction.

\section{CONCLUSION}

In this paper, a new approximate formulation for scattering by a very thin homogeneous planar dielectric structures is presented. This formulation is derived based on a VIE for the induced polarization current. Using the spectral domain representation of the free-space dyadic Green's function, the polarization current inside an arbitrarily shaped thin dielectric disk is expressed in a closed-form equation in the spectral domain. Apart from the thin dielectric approximation, the formulation is obtained by replacing a shape-related convolution with the a simple multiplication which is shown to be valid for small $t\left(\varepsilon_{r}-1\right) / \lambda$ where $t$ is the thickness and $\varepsilon_{r}$ is the dielectric constant of the dielectric sheet. The validity of the formulation is examined using several structures. For an infinite dielectric slab, it is shown that the exact solution can be obtained using thin slab approximation. For 2-D and 3-D problems, thin dielectric half-planes and finite strips, and circular and square disks of wide range of size at different incidence angles are investigated, respectively. For thin dielectric half-planes, a closed-form solution for backscattering is formulated for edge-on incidence cases and compared with known solutions for resistive half-planes. For other geometries, scattered far-field behavior such as forward-, back-, and bistaicscattering are compared with results computed by MoM and excellent agreements are obtained.

\section{REFERENCES}

[1] T. B. A. Senior and J. L. Volakis, Approximate Boundary Conditions in Electromagnetics. London, U.K.: Institution of Electrical Engineers, 1995.

[2] J. J. Bowman, T. B. A. Senior, P. L. E. Uslenghi, and J. S. Asvestas, Electromagnetic and Acoustic Scattering by Simple Shapes. New York: Hemisphere, 1987.

[3] Y. C. Lin and K. Sarabandi, "Monte Carlo coherent scattering model for forest canopies using fractal-generated trees," IEEE Trans. Geoscie. Remote Sensing, vol. 37, no. 1, pp. 440-451, Jan. 1999.

[4] I. Koh and K. Sarabandi, "Polarimetric channel characterization of foliage for performance assessment of GPS receiver under tree canopies," IEEE Trans. Antennas Propag., vol. 50, no. 5, pp. 713-726, May 2002.

[5] I. Koh, F. Wang, and K. Sarabandi, "Estimation of coherent field attenuation through dense foliage including multiple scattering," IEEE Trans. Geosci. Remote Sensing, vol. 41, no. 5, pp. 1132-1135, May 2003.

[6] R. F. Harrington, Time-Harmonic Electromagnetic Fields. New York: McGraw-Hill, 1961.

[7] J. J. H. Wang, Generalized Moment Methods in Electromagnetics: Formulation and Computer Solution of Integral Equations. New York: Wiley, 1991.

[8] L. Tsang, J. Kong, and R. T. Shin, Theory of Microwave Remote Sensing. New York: Wiley, 1985.
[9] F. T. Ulaby, R. K. Moore, and A. K. Fung, Microwave Remote Sensing Active and Passive. Norwood, MA: Artech House, 1982.

[10] K. Sarabandi and I. Koh, "Effect of canopy-air interface roughness on HF-UHF wave propagation in forest," IEEE Trans. Antennas Propag., vol. 50, no. 2, pp. 101-110, Feb. 2002.

[11] K. Sarabandi and T. Chiu, "Electromagnetic scattering from slightly rough surfaces with inhomogeneous dielectric profiles," IEEE Trans. Antennas Propag., vol. 45, no. 9, pp. 1419-1430, Sep. 1997.

[12] J. H. Richmond, "Scattering by thin dielectric strips," IEEE Trans. Antennas Propag., vol. AP-33, no. 1, pp. 64-68, Jan. 1985.

[13] T. B. A. Senior, "Backscattering from resistive strips," IEEE Trans. Antennas Propag., vol. AP-27, no. 6, pp. 808-813, Nov. 1979.

[14] R. Schiffer and K. O. Thielheim, "Light scattering by dielectric needles and disks," J. Appl. Physics, vol. 50, no. 4, pp. 2476-2483, Apr. 1979.

Il-Suek Koh was born in Korea. He received the B.S. and M.S. degrees in electronics engineering from Yonsei University, Seoul, Korea, in 1992 and 1994, respectively, and the Ph.D. degree from The University of Michigan at Ann Arbor, in 2002.

In 1994, he joined LG Electronics Ltd., Seoul, as a Research Engineer. Currently, he is with Inha University as a Full Lecturer. His research interests are communication channel modeling, and numerical and analytical techniques.

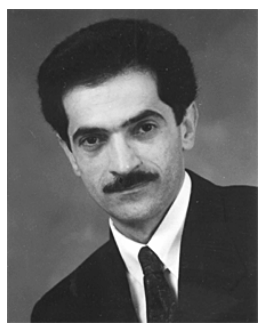

Kamal Sarabandi (S'87-M'90-SM'92-F'00) received the B.S. degree in electrical engineering from Sharif University of Technology, Tehran, Iran, in 1980, the M.S. degree in electrical engineering/mathematics, and the Ph.D. degree in electrical engineering from The University of Michigan-Ann Arbor, in 1986 and 1989, respectively.

He is Director of the Radiation Laboratory and a Professor in the Department of Electrical Engineering and Computer Science, The University of Michigan-Ann Arbor. He has 20 years of experience with wave propagation in random media, communication channel modeling, microwave sensors, and radar systems and is leading a large research group consisting of four research scientists, 12 Ph.D. students, and two M.S. students. Over the past ten years he has graduated $20 \mathrm{Ph} . \mathrm{D}$. students. He has served as the Principal Investigator on many projects sponsored by NASA, JPL, ARO, ONR, ARL, NSF, DARPA, and numerous industries. He has published many book chapters and more than 115 papers in refereed journals on electromagnetic scattering, random media modeling, wave propagation, antennas, microwave measurement techniques, radar calibration, inverse scattering problems, and microwave sensors. He has also had more than 230 papers and invited presentations in many national and international conferences and symposia on similar subjects. His research areas of interest include microwave and millimeter-wave radar remote sensing, electromagnetic wave propagation, and antenna miniaturization.

Dr. Sarabandi is a Member of the International Scientific Radio Union (URSI) Commission F and of The Electromagnetic Academy. He received the Henry Russel Award from the Regent of The University of Michigan-Ann Arbor (the highest honor the University of Michigan bestows on a faculty member at the assistant or associate level). He received a 1996 Teaching Excellence Award from the Department of Electrical Engineering and Computer Science and a 1999 GAAC Distinguished Lecturer Award from the German Federal Ministry for Education, Science, and Technology, given to about ten individuals worldwide in all areas of engineering, science, medicine, and law. He also received the 2003/2004 College of Engineering Research Excellence Award, The University of Michigan-Ann Arbor. In the past several years, joint papers presented by his students at a number of symposia (IEEE AP'95,'97,'00,'01,'03 IEEE IGARSS'99,'02, IEEE MTTS'01) have received student prize paper awards. He is a Vice President of the IEEE Geoscience and Remote Sensing Society (GRSS), a past Chairman of the Awards Committee of the IEEE GRSS from 1998 to 2002, and a Member of the IEEE Technical Activities Board Awards Committee from 2000 to 2002. He is an Associate Editor of the IEEE TRANSACTIONS ON ANTENNAS AND PROPAGation and the IEEE SENSORS JOURNAL. He is listed in American Men \& Women of Science, Who's Who in America, and Who's Who in Electromagnetics. 